\title{
Effects of Newer Antidiabetic Drugs on Endothelial Function and Arterial Stiffness: A Systematic Review and Meta-Analysis
}

\author{
Konstantinos Batzias, ${ }^{1}$ Alexios S. Antonopoulos, ${ }^{1}$ Evangelos Oikonomou ${ }^{1 D},{ }^{1}$ \\ Gerasimos Siasos, ${ }^{1}$ Evanthia Bletsa, ${ }^{1}$ Panagiota K. Stampouloglou, ${ }^{1}$ \\ Chara-Vasiliki Mistakidi, ${ }^{1}$ Marina Noutsou, ${ }^{2}$ Niki Katsiki, ${ }^{3}$ Periklis Karopoulos, \\ Georgios Charalambous, ${ }^{1}$ Anastasia Thanopoulou, ${ }^{2}$ Nicholas Tentolouris $\mathbb{D},{ }^{4}$ \\ and Dimitris Tousoulis ${ }^{1}$ \\ ${ }^{1}$ 1st Department of Cardiology, Hippokration Hospital, National and Kapodistrian University of Athens Medical School, \\ Athens, Greece \\ ${ }^{2}$ Diabetes Center, 2nd Department of Internal Medicine, Medical School, National and Kapodistrian University of Athens, \\ Hippokration General Hospital of Athens, Athens, Greece \\ ${ }^{3}$ Second Department of Internal Medicine, Hippokration University Hospital, Aristotle University of Thessaloniki, \\ Thessaloniki, Greece \\ ${ }^{4}$ First Department of Propaedeutic and Internal Medicine, Division of Diabetes, Laiko University Hospital, National and \\ Kapodistrian University of Athens Medical School, Athens, Greece
}

Correspondence should be addressed to Evangelos Oikonomou; boikono@gmail.com

Received 11 July 2018; Revised 25 October 2018; Accepted 1 November 2018; Published 4 December 2018

Academic Editor: Yoshifumi Saisho

Copyright (C) 2018 Konstantinos Batzias et al. This is an open access article distributed under the Creative Commons Attribution License, which permits unrestricted use, distribution, and reproduction in any medium, provided the original work is properly cited.

\begin{abstract}
Background. Newer antidiabetic drugs, i.e., dipeptidyl peptidase-4 (DPP-4) inhibitors, sodium-glucose cotransporter-2 (SGLT-2) inhibitors, and glucagon-like peptide-1 receptor agonists (GLP-1 RAs) may exert distinct cardiovascular effects. We sought to explore their impact on vascular function. Methods. Published literature was systematically searched up to January 2018 for clinical studies assessing the effects of DPP-4 inhibitors, GLP-1 RAs, and SGLT-2 inhibitors on endothelial function and arterial stiffness, assessed by flow-mediated dilation (FMD) of the brachial artery and pulse wave velocity (PWV), respectively. For each eligible study, we used the mean difference (MD) with 95\% confidence intervals (CIs) for FMD and PWV. The pooled MD for FMD and PWV were calculated by using a random-effect model. The presence of heterogeneity among studies was evaluated by the $I^{2}$ statistic. Results. A total of 26 eligible studies $(n=668$ patients) were included in the present meta-analysis. Among newer antidiabetic drugs, only SGLT-2 inhibitors significantly improved FMD (pooled MD 1.14\%, 95\% CI: 0.18 to 1.73, $p=0.016$ ), but not DPP-4 inhibitors (pooled $\mathrm{MD}=0.86 \%, 95 \% \mathrm{CI}$ : -0.15 to $1.86, p=0.095$ ) or GLP-1 RA (pooled MD $=2.37 \%, 95 \% \mathrm{CI}$ : -0.51 to $5.25, p=0.107$ ). Both GLP-1 RA (pooled $\mathrm{MD}=-1.97,95 \% \mathrm{CI}:-2.65$ to $-1.30, p<0.001$ ) and, to a lesser extent, DPP-4 inhibitors (pooled $\mathrm{MD}=-0.18,95 \% \mathrm{CI}:-0.30$ to $-0.07, p=0.002$ ) significantly decreased PWV. Conclusions. Newer antidiabetic drugs differentially affect endothelial function and arterial stiffness, as assessed by FMD and PWV, respectively. These findings could explain the distinct effects of these drugs on cardiovascular risk of patients with type 2 diabetes.
\end{abstract}

\section{Introduction}

Type 2 diabetes (T2D) is a chronic disease affecting $8.3 \%$ of the adult population worldwide, with a rising prevalence that renders its tackling a global challenge [1]. Patients with T2D are at high cardiovascular disease (CVD) risk $[2,3]$ and are characterized by micro- and macrovascular dysfunction which is of multifactorial origin $[4,5]$.

The safety and effects of newly licensed antidiabetic drugs on the cardiovascular system represent important clinical 
issues $[6,7]$. Recent evidence from clinical trials suggests that newer antidiabetic drugs can not only exert glycemiclowering properties but also decrease CVD risk $[8,9]$. In this context, sodium-glucose cotransporter-2 (SGLT-2) inhibitors, i.e., empagliflozin in the Empagliflozin Cardiovascular Outcome Event Trial in Type 2 Diabetes Mellitus PatientsRemoving Excess Glucose (EMPA-REG OUTCOME) study [8] and canagliflozin in the Canagliflozin Cardiovascular Assessment Study [10], significantly reduced the rates of CVD events, hospitalization for heart failure (HF), CVD, and total mortality, as well as improved kidney function in T2D patients with established CVD. Similar beneficial effects were reported for liraglutide, an once-daily glucagon-like peptide-1 receptor agonist (GLP-1 RA), and for semaglutide, an once-weekly GLP-1 RA, both of which reduced CVD morbidity and mortality (but not hospitalization for HF) in T2D patients with established CVD, in the Liraglutide Effect and Action in Diabetes: Evaluation of Cardiovascular Outcome Results (LEADER) trial [9] and the Trial to Evaluate Cardiovascular and Other Long-term Outcomes with Semaglutide in Subjects with Type 2 Diabetes (SUSTAIN-6) [11], respectively. In contrast, lixisenatide once daily and exenatide once weekly did not affect CVD risk in the Evaluation of Lixisenatide in Acute Coronary Syndrome (ELIXA) trial [12] and the Exenatide Study of Cardiovascular Event Lowering (EXSCEL) [13], respectively. Furthermore, dipeptidyl peptidase-4 (DPP-4) inhibitors seem to exert neutral effects on CVD risk as shown for alogliptin in the Examination of Cardiovascular Outcomes with Alogliptin versus Standard of Care (EXAMINE) trial [14] and for sitagliptin in the Trial Evaluating Cardiovascular Outcomes with Sitagliptin (TECOS) [15]. Saxagliptin was reported to increase the rate of hospitalization for HF [16] in the Saxagliptin Assessment of Vascular Outcomes Recorded in Patients with Diabetes Mellitus (SAVOR)-Thrombolysis in Myocardial Infarction (TIMI) 53 trial. Despite this evidence provided by large randomized clinical trials, the mechanisms by which antidiabetic drugs can affect CVD risk remain not entirely clear.

Vascular dysfunction is one of the initial steps in the atherosclerotic process $[17,18]$. Endothelial function and arterial stiffness $[17,19]$ are two widely used indices of vascular function, which both offer prognostic information on the risk of CVD events in T2D patients [19]. Improvement of these indices represents one of the mechanisms by which drugs with established CVD benefits, such as statins, exert their effects $[20,21]$. Currently, it remains unknown how newer antidiabetic drugs may affect vascular function as studies have yielded conflicting results.

We conducted a systematic review of the literature, followed by a meta-analysis, to investigate the effects of newer antidiabetic drugs, i.e., DPP-4 inhibitors, GLP-1 RAs, and SGLT-2 inhibitors, on vascular function as assessed by flow-mediated dilation (FMD) of the brachial artery and pulse wave velocity (PWV).

\section{Patients and Methods}

2.1. Literature Search. Eligible studies evaluating the effects of DPP-4 inhibitors, GLP-1 RAs, and SGLT-2 inhibitors on
FMD and PWV were drawn from a systematic review of the English literature in the MEDLINE and Web of Science databases up to 31 January 2018. The medical terms (MeSH) used were the following: sodium-glucose cotransporter-2 OR SGLT2 OR empagliflozin OR canagliflozin OR dapagliflozin OR DPP-4 OR dipeptidyl peptidase-4 inhibitors OR sitagliptin OR saxagliptin OR vildagliptin OR linagliptin OR gemigliptin OR canagliptin OR teneligliptin OR alogliptin OR trelagliptin OR omarigliptin OR evogliptin OR dutogliptin OR GLP-1 OR glucagon-like peptide-1 OR exenatide OR lixisenatide OR dulaglutide $O R$ liraglutide $O R$ semaglutide AND endothelial function OR arterial stiffness OR flowmediated dilation OR pulse wave velocity. Studies were also identified from searching the references of published articles. The PRISMA flow chart for the study is presented in Figure 1.

2.2. Study Eligibility. Studies were eligible if they were fulllength publications in peer-reviewed journals reporting on (a) endothelium-dependent vasodilatory response by FMD and/or (b) arterial stiffness assessed by carotid-femoral, carotid-radial, or brachial-ankle PWV. Studies need also to be either double-blind, placebo-controlled, randomized clinical trials or observational studies assessing the outcomes of interest before and after treatment with a newer antidiabetic drug. No restriction criteria were imposed with regard to the size of the population studied. Analysis did not include studies assessing endothelial function or arterial stiffness by other markers. Only human studies were included in the analysis, whereas review articles were excluded.

2.3. Extraction of Data. Literature search, selection of studies, and extraction of data were performed independently by two investigators (KB and $\mathrm{AA})$. Means and standard deviations (SD) of FMD and PWV as well as their changes following drug treatment were recorded from cumulative published data. In studies reporting standard error, SD was calculated using the equation $\mathrm{SD}=$ standard error $* \sqrt{ } n$. In studies reporting median and interquartile range, mean and SD were calculated and used in further analyses [22]. In those studies where extraction of cumulative statistics could not be reliably performed based on the full-length publication, a communication with the corresponding author was attempted to provide summary statistics [23-27].

2.4. Statistical Analysis. For each eligible study, we used the mean difference (MD) with 95\% confidence intervals (CIs) for endothelium-dependent vasodilation and arterial stiffness as summary statistics (MD pre- and posttreatment). The difference in mean \pm SD for FMD and PWV was included in the quantitative synthesis to explore the pooled MD after treatment with any of the studied antidiabetic drugs; the results were presented in respective forest plots. Subgroup analysis was performed for different drug classes. The presence of heterogeneity was evaluated by the $I^{2}$ statistic. A randomeffect model was used to obtain pooled MD and 95\% CIs. Results were considered statistically significant at two-tailed $p$ value $<0.05$. The findings of the meta-analysis were also confirmed in leave-one-out sensitivity analysis. The quality of published studies was assessed by the modified version of 

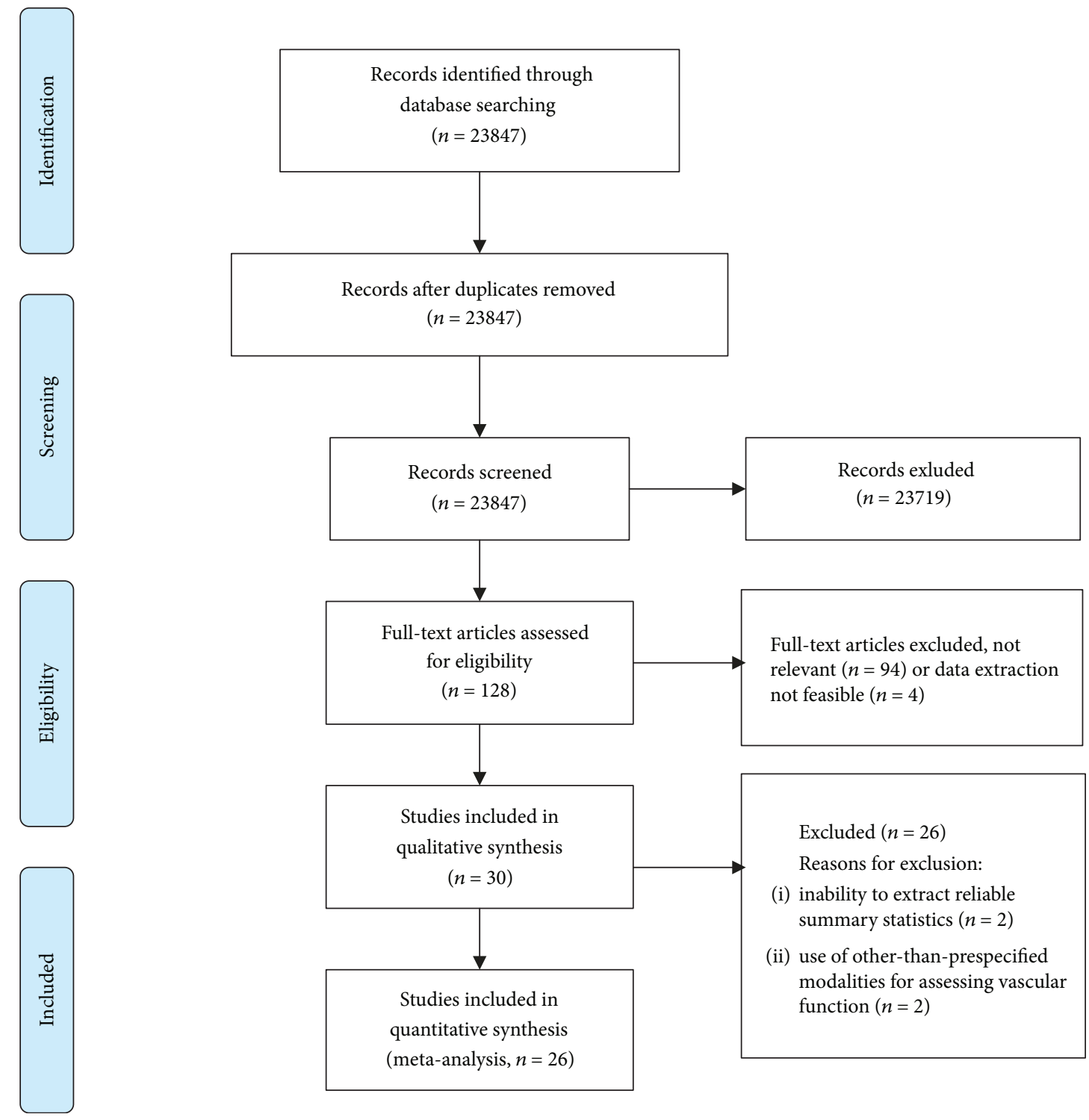

FIgURE 1: Study flow chart.

the Downs and Black questionnaire [28]. The effect of publication bias on pooled estimates was explored by the Duval and Tweedie nonparametric "trim and fill" method and the construction of relevant funnel plots. All statistical analyses were performed by STATA software version 13.0 (StataCorp LLC, College Station, Texas, US).

\section{Results}

3.1. Qualitative Summary. The literature search identified 30 studies for potential inclusion in the present meta-analysis. Certain identified studies that were originally included in the qualitative synthesis had to be excluded from the quantitative synthesis due to not fulfilling the eligibility criteria. One study was excluded because it assessed endothelial function by reactive hyperemia peripheral arterial tonometry [29] and one because it used a $24 \mathrm{~h}$ approach to measure PWV [30]. Also, two studies had to be excluded due to inability to extract reliable summary statistics for the outcomes of interest $[26,27]$ (Figure 1). Therefore, a total of 26 studies ( $n=668$ patients) were finally included in the quantitative synthesis. The details of the individual studies included in the meta-analysis are summarized in Table 1.

All studies were published since 2012. The sample sizes of the studies ranged from 10 to 51 patients. The mean followup time was 152 days after initiation of treatment. The majority of studies were randomized controlled trials [23$25,31-48]$, but some of them were uncontrolled or singlearm observational studies [49-53]. All studies performed the ultrasound-based technique to assess brachial artery FMD and PWV to assess arterial stiffness.

\subsection{Quantitative Synthesis}

3.2.1. Effects of Newer Antidiabetic Drugs on Endothelial Function. The effects of newer antidiabetic drugs on FMD 
TABLE 1: Summary characteristics of studies included in the analysis.

\begin{tabular}{|c|c|c|c|c|c|c|c|c|c|}
\hline \multirow{2}{*}{ Author } & \multirow{2}{*}{ Class } & \multirow{2}{*}{ Agent } & \multirow{2}{*}{ Study design } & \multirow{2}{*}{$N$} & \multirow{2}{*}{$\begin{array}{c}\text { Duration } \\
\text { (days) }\end{array}$} & \multicolumn{2}{|c|}{ FMD (\%) } & \multicolumn{2}{|c|}{$\mathrm{PWV}(\mathrm{m} / \mathrm{s})$} \\
\hline & & & & & & Baseline & Post & Baseline & Post \\
\hline \multirow{2}{*}{ Ayaori et al. [31] } & \multirow{2}{*}{ DPP-4i } & Sitagliptin & RCT & 42 & 42 & $7.2 \pm 5.9$ & $4.4 \pm 5.9$ & & \\
\hline & & Alogliptin & & & & $6.9 \pm 6.3$ & $4.4 \pm 6.2$ & & \\
\hline Baltzis et al. [32] & DPP-4i & Linagliptin & RCT & 19 & 84 & $6.5 \pm 2.1$ & $7.2 \pm 2.5$ & & \\
\hline de Boer et al. [33] & DPP-4i & Linagliptin & RCT & 22 & 182 & & & $8.7 \pm 1.6$ & $8.3 \pm 1.3$ \\
\hline Dell'Oro et al. [34] & DPP-4i & Saxagliptin & RCT & 16 & 360 & $3.6 \pm 0.3$ & $7.4 \pm 0.8$ & & \\
\hline Duvnjak et al. [49] & DPP-4i & Sitagliptin/vildagliptin & Open label (NR) & 51 & 90 & & & $8.6 \pm 0.3$ & $8.4 \pm 0.3$ \\
\hline Gurkan et al. [35] & GLP-1 RA & Exenatide & RCT & 17 & 182 & $6.4 \pm 5.7$ & $8.6 \pm 4.7$ & & \\
\hline Hong et al. [50] & GLP-1 RA & Exenatide & Single arm (NR) & 32 & 90 & & & $7.2 \pm 2.2$ & $5.1 \pm 0.1$ \\
\hline Hopkins et al. [51] & GLP-1 RA & Exenatide/liraglutide & Single arm (NR) & 11 & 180 & $6.2 \pm 2.3$ & $5.1 \pm 2.7$ & & \\
\hline Ida et al. [52] & DPP-4i & Trelagliptin & Single arm (NR) & 27 & 84 & $2.4 \pm 2.7$ & $2.7 \pm 3.8$ & $16.3 \pm 2.4$ & $15.6 \pm 2.2^{*}$ \\
\hline Irace et al. [36] & GLP-1 RA & Exenatide & RCT & 10 & 112 & $1.6 \pm 2.9$ & $9.1 \pm 3.6$ & & \\
\hline Nakamura et al. [24] & DPP-4i & Sitagliptin & RCT & 24 & 90 & $5.4 \pm 2.3$ & $6.2 \pm 2.0$ & & \\
\hline Kim et al. [37] & DPP-4i & Vildagliptin & RCT & 17 & 84 & $9.4 \pm 5.0$ & $7.9 \pm 4.3$ & & \\
\hline Kitao et al. [38] & DPP-4i & Vildagliptin & RCT & 48 & 84 & $5.5 \pm 2.0$ & $5.1 \pm 2.3$ & & \\
\hline Kubota et al. [53] & DPP-4i & Sitagliptin & Single arm (NR) & 40 & 90 & $4.1 \pm 1.5$ & $5.1 \pm 1.6$ & & \\
\hline Lambadiari et al. [39] & GLP-1 RA & Liraglutide & RCT & 30 & 180 & $8.9 \pm 3.0$ & $13.2 \pm 6.0$ & $11.8 \pm 2.5$ & $10.3 \pm 3.3$ \\
\hline Leung et al. [40] & DPP-4i & Sitagliptin/vildagliptin & RCT & 25 & 365 & $2.4 \pm 1.6$ & $7.3 \pm 1.6$ & & \\
\hline Li et al. [41] & DPP-4i & Saxagliptin & RCT & 14 & 84 & $9.3 \pm 4.7$ & $14.3 \pm 4.3$ & & \\
\hline Nomoto et al. [42] & DPP-4i & Sitagliptin & RCT & 48 & 182 & $5.6 \pm 2.8$ & $5.6 \pm 2.8$ & & \\
\hline Nomoto et al. [43] & GLP-1 RA & Liraglutide & RCT & 16 & 98 & $6.0 \pm 2.6$ & $5.6 \pm 1.6$ & & \\
\hline Shigiyama et al. [44] & SGLT-2i & Dapagliflozin & RCT & 37 & 112 & $4.8 \pm 1.9$ & $5.7 \pm 2.1$ & & \\
\hline Shigiyama et al. [45] & DPP-4i & Linagliptin & RCT & 29 & 112 & $4.9 \pm 2.7$ & $6.3 \pm 2.7$ & & \\
\hline Solini et al. [46] & SGLT-2i & Dapagliflozin & RCT & 16 & 2 & $2.8 \pm 2.3$ & $4.0 \pm 2.1$ & $10.1 \pm 1.6$ & $8.9 \pm 1.6$ \\
\hline Suzuki et al. [25] & DPP-4i & Sitagliptin & RCT & 12 & 90 & $3.7 \pm 2.3$ & $5.4 \pm 2.2$ & & \\
\hline Maruhashi et al. [23] & DPP-4i & Sitagliptin & RCT & 17 & 720 & $4.3 \pm 2.6$ & $4.4 \pm 2.3$ & & \\
\hline Widlansky et al. [47] & DPP-4i & Saxagliptin & RCT & 16 & 56 & $5.6 \pm 2.3$ & $5.8 \pm 2.3$ & & \\
\hline Zografou et al. [48] & DPP-4i & Vildagliptin & RCT & 32 & 180 & & & $8.6 \pm 2.1$ & $8.3 \pm 1.5$ \\
\hline
\end{tabular}

DPP-4i: dipeptidyl peptidase-4 inhibitor; FMD: flow-mediated dilation; GLP-1 RA: glucagon-like peptide-1 receptor agonist; NR: nonrandomized; RCT: randomized clinical trial; SGLT-2i: sodium-glucose cotransporter-2 inhibitor; PWV: pulse wave velocity. $N$ refers to the active treatment group. The full list of references of the studies included in the table is provided in the supplementary material. * Measured as brachial-ankle PWV.

are summarized in Figure 2. Overall, 16 studies investigated the effects of DPP-4 inhibitors on FMD. In the pooled meta-analysis, the effects of DPP-4 inhibitors on FMD was not statistically significant (pooled $\mathrm{MD}=0.86 \%, 95 \% \mathrm{CI}$ : -0.15 to $1.86, p=0.095)$. But there was significant heterogeneity between studies $\left(I^{2}=87.8 \%, p<0.0001\right)$. In leaveone-out sensitivity analysis, the results were unchanged (Table 2); only by excluding the study of Ayaori et al. [31] was a significant effect of DPP-4 on FMD observed (pooled $\mathrm{MD}=1.25 \%, 95 \% \mathrm{CI}: 0.24$ to $2.27, p=0.015$ ), but heterogeneity among studies remained significant. Even within the group of DPP-4 inhibitors, no specific agent was associated with significant improvements in FMD.

For the effect of GLP-1 RAs (liraglutide and exenatide) on FMD (5 studies; $n=84$ patients), the pooled estimate effect was not significant (pooled $\mathrm{MD}=2.38 \%$, 95\% CI: -0.51 to $5.25, p=0.107)$. Heterogeneity among studies was significant $\left(I^{2}=88.4 \%, p<0.001\right)$. In leave-one-out sensitivity analysis, the results were unchanged and the heterogeneity among studies remained significant.
Only 2 eligible studies ( $n=53$ patients) evaluated the effects of SGLT-2 inhibitors on FMD. Dapagliflozin was the only SGLT-2 inhibitor used in these studies. SGLT-2 inhibitors increased FMD significantly (pooled $\mathrm{MD}=0.95 \%, 95 \%$ CI: 0.18 to $1.73, p=0.016$ ).

In metaregression analysis, the mean difference in FMD was not associated with the size of the study $(b=-0.033, p=0.489)$, the duration of intervention $(b=0.002, p=0.460)$, the age $(b=-0.162, p=0.163)$ or the sex (for males: $b=0.004, p=0.931$ ) of participants enrolled.

\subsubsection{Effects of Newer Antidiabetic Drugs on Arterial Stiffness.} The effects of newer antidiabetic drugs on PWV are summarized in Figure 3. Marked heterogeneity was observed among analyzed studies $\left(I^{2}=80.7 \%, p<0.001\right)$. A total of 4 studies ( $n=132$ patients) investigated the effects of DPP-4 inhibitors on arterial stiffness, which were associated with a significant reduction in $\mathrm{PWV}$ (pooled $\mathrm{MD}=-0.18,95 \% \mathrm{CI}:-0.30$ to -0.07, $p=0.002)$. A similar effect was identified for GLP-1 RAs ( 2 studies; $n=62$ patients), which were associated with 


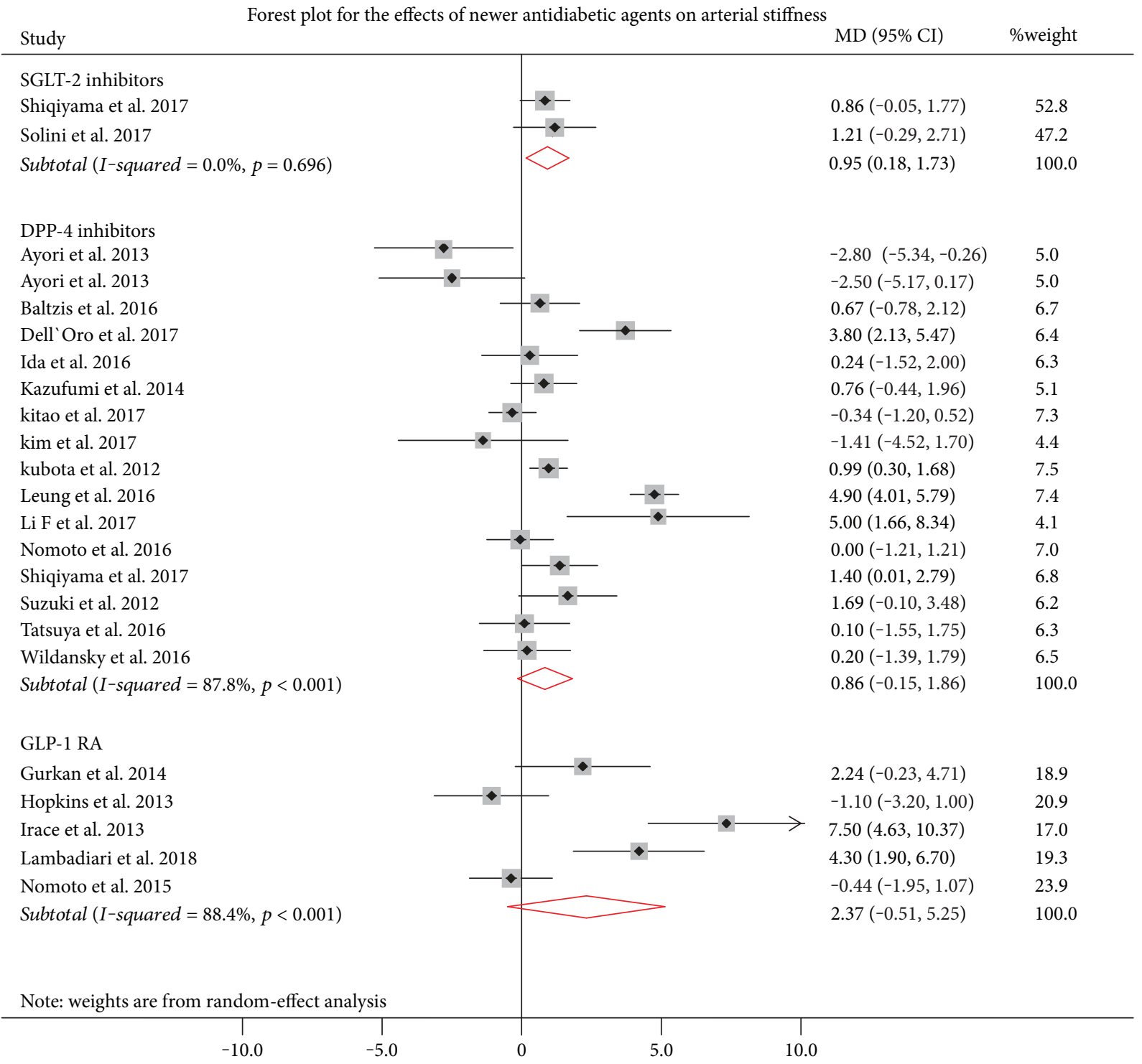

FIGURE 2: Effects of newer antidiabetic drugs on endothelial function. Squares indicate the mean difference (MD) and the respective 95\% confidence intervals in flow-mediated dilatation (FMD) before/after treatment from eligible studies. The size of the squares corresponds to the weight of each study. The diamonds and their width represent the pooled MD and the 95\% CI, respectively. DPP-4=DPP-4 inhibitors, SGLT-2 inhibitors, and GLP-1 RAs and defines them under the figure. DPP-4: dipeptidyl peptidase-4; GLP-1 RAs: glucagonlike peptide-1 receptor agonists; SGLT-2: sodium-glucose cotransporter-2.

a significant reduction in $\mathrm{PWV}$ (pooled $\mathrm{MD}=-1.97,95 \% \mathrm{CI}$ : -2.65 to $-1.30, p<0.001)$. For SGLT-2 inhibitors, only one study reported a reduction in $\mathrm{PWV}$, and therefore, no safe conclusions can be drawn.

3.3. Quality Assessment of Included Studies and Publication Bias. The quality of published studies was assessed by the modified Downs and Black checklist [28]. The quality of published studies is considered moderate with a mean modified Downs and Black score of 23.1. To explore publication bias, we constructed a funnel plot of published studies for the effect size of antidiabetic drugs on the primary endpoint of our study, i.e., endothelial function assessed by FMD. The funnel plot was symmetric, suggesting the absence of publication bias (Figure 4).

\section{Discussion}

In the present systematic review, we sought to explore the effects of newer antidiabetic drugs, namely, SGLT-2 inhibitors, DPP-4 inhibitors, and GLP-1 RAs, on vascular function. We hypothesized that the distinct profile of each antidiabetic drug class could be also associated with differences in their vascular effects. The systematic review of the published literature showed that evidence in this field is modest, based mainly on small randomized clinical trials with significant heterogeneity. In this context, published studies supported a beneficial effect of SGLT-2 on FMD, which seems to not be shared by GLP-1 RAs or DPP-4 inhibitors. Accordingly, evidence suggested a reduction in PWV by both DPP-4 inhibitors and GLP-1 RAs. These findings are potentially 
TABLE 2: Leave-one-out sensitivity analysis for the effects of newer antidiabetics on endothelial function.

\begin{tabular}{|c|c|c|c|}
\hline Study excluded & $\mathrm{MD}(95 \% \mathrm{CI})$ & $p$ value & Heterogeneity $\left(I^{2}\right)$ \\
\hline \multicolumn{4}{|l|}{ DPP-4 inhibitors } \\
\hline Ayaori et al. [31] & $1.253(0.242$ to 2.265$)$ & $p=0.015$ & $87.7 \%, p<0.001$ \\
\hline Baltzis et al. [32] & $0.866(-0.205$ to 1.936$)$ & $p=0.113$ & $88.6 \%, p<0.001$ \\
\hline Dell'Oro et al. [34] & $0.658(-0.365$ to 1.682$)$ & $p=0.207$ & $87.8 \%, p<0.001$ \\
\hline Ida et al. [52] & $0.897(-0.151$ to 1.945$)$ & $p=0.095$ & $88.5 \%, p<0.001$ \\
\hline Nakamura et al. [24] & $0.858(-0.226$ to 1.943$)$ & $p=0.121$ & $88.6 \%, p<0.001$ \\
\hline Kim et al. [37] & $0.962(-0.066$ to 1.990$)$ & $p=0.067$ & $88.3 \%, p<0.001$ \\
\hline Kitao et al. [38] & $0.948(-0.115$ to 2.011$)$ & $p=0.080$ & $87.1 \%, p<0.001$ \\
\hline Kubota et al. [53] & $0.832(-0.329$ to 1.993$)$ & $p=0.160$ & $88.6 \%, p<0.001$ \\
\hline Leung et al. [40] & $0.566(-0.130$ to 1.261$)$ & $p=0.111$ & $68.6 \%, p<0.001$ \\
\hline Li et al. [41] & $0.679(-0.337$ to 1.695$)$ & $p=0.190$ & $88.1 \%, p<0.001$ \\
\hline Nomoto et al. [42] & $0.917(-0.153$ to 1.986$)$ & $p=0.093$ & $88.2 \%, p<0.001$ \\
\hline Shigiyama et al. [44] & $0.813(-0.262$ to 1.888$)$ & $p=0.138$ & $88.6 \%, p<0.001$ \\
\hline Suzuki et al. [25] & $0.798(-0.261$ to 1.858$)$ & $p=0.140$ & $88.6 \%, p<0.001$ \\
\hline Maruhashi et al. [23] & $0.906(-0.152$ to 1.964$)$ & $p=0.093$ & $88.4 \%, p<0.001$ \\
\hline Widlansky et al. [47] & $0.899(-0.161$ to 1.960$)$ & $p=0.097$ & $88.5 \%, p<0.001$ \\
\hline \multicolumn{4}{|l|}{ GLP-1 RA } \\
\hline Gurkan et al. [35] & $2.435(-1.177$ to 6.047$)$ & $p=0.186$ & $91.2 \%, p<0.001$ \\
\hline Hopkins et al. [51] & $3.275(-0.132$ to 6.682$)$ & $p=0.060$ & $89.2 \%, p<0.001$ \\
\hline Irace et al. [36] & $1.141(-1.194$ to 3.476$)$ & $p=0.338$ & $80.2 \%, p=0.001$ \\
\hline Lambadiari et al. [39] & $1.902(-1.399$ to 5.203$)$ & $p=0.259$ & $89.3 \%, p<0.001$ \\
\hline Nomoto et al. [42] & $3.155(-0.376$ to 6.687$)$ & $p=0.080$ & $88.1 \%, p<0.001$ \\
\hline
\end{tabular}

DPP-4: dipeptidyl peptidase-4 inhibitors; GLP-1 RA: glucagon-like peptide-1 receptor agonists.

important as they suggest a different impact of newer antidiabetic drugs on vascular function, which could be linked with their distinct effects on CVD risk. However, these results should be interpreted with caution given the modest quality of evidence in the published literature and the significant heterogeneity between studies.

DPP-4 inhibitors are an antidiabetic drug class on which there is abundant clinical experience, since they have been marketed for over a decade (since 2006). Large randomized clinical trials in the field are consistent in their findings and support a neutral effect of DPP-4 inhibitors on CVD outcomes. However, there is evidence that saxagliptin may increase the risk for HF hospitalization [16]. Recent metaanalyses have also found that DPP-4 inhibitors do not affect the risk for CVD mortality and stroke [54]. Data from the small clinical studies included in the present meta-analysis indicate a marginal effect of DPP-4 drugs on FMD and a significant reduction in PWV. These small effects could be related to the glucose-lowering properties of DPP-4 inhibition but may not be commonly shared by all agents of the DPP-4 drug class. This is an interest finding which (a) confirms the safety profile of this drug class and (b) may explain the neutral effect of certain DPP-4 drugs on CVD outcomes.

For SGLT-2 inhibitors, evidence suggests a beneficial effect of these agents on CVD risk and mortality in T2D patients with established CVD. Evidence from randomized clinical trials suggests that empagliflozin and canagliflozin significantly reduce the CVD morbidity, all-cause mortality, and CVD mortality as well as HF hospitalization and nephropathy development or progression [8]. Similar effects have also been reported for canagliflozin [10]. These benefits could be related to glucose-lowering as well as to reductions in blood pressure, weight, and serum uric acid and to improvements in oxidative stress, glomerular hyperfiltration, albuminuria, arterial stiffness, plasma lipids, sympathetic nervous system activity, myocardial oxygen consumption, and cardiac workload [55]. Our findings complement these SGLT-2 inhibitor actions, suggesting also a significant improvement in FMD. Concerning the impact of SGLT-2 inhibitors on arterial wave velocity, it should be noted that despite the ample evidence on the impact of this class of drugs on natriuresis and blood pressure $[8,10]$, more data are required to assess the effects of these drugs on PWV, as there are only few published data on this topic [46].

Our findings also agree with the published evidence from large randomized clinical trials on the effects of liraglutide [9] and semaglutide [11] on CVD outcomes in T2D patients. In the present meta-analysis, GLP-1 RAs significantly decreased PWV in T2D patients, although they did not affect FMD. Since the effects of these drugs on CVD risk is still debatable $[12,56]$, it remains to be seen whether their impact on vascular function may play a role in determining their CVD effects. 


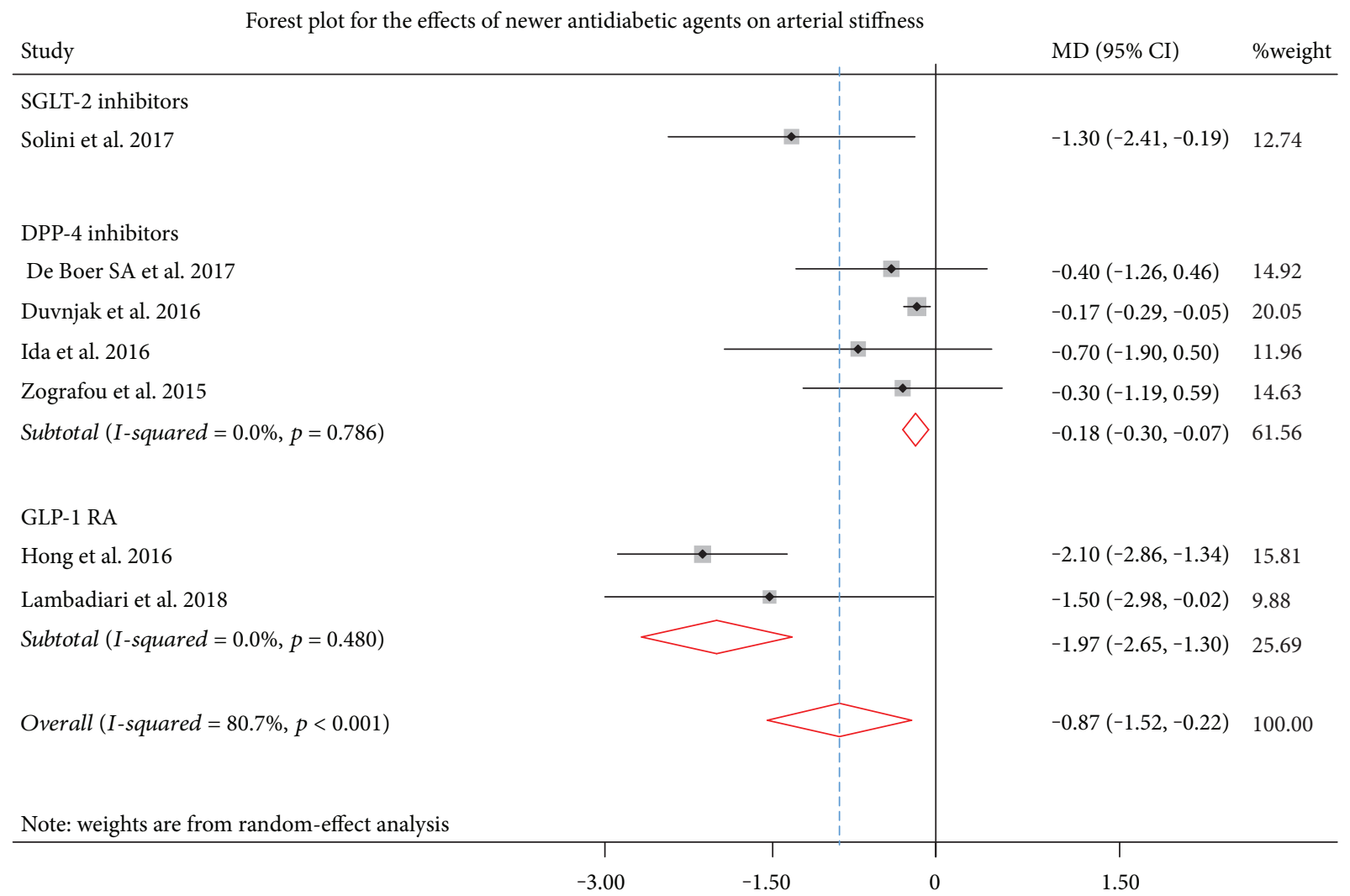

FIGURE 3: Effects of newer antidiabetic drugs on arterial stiffness. Squares indicate the mean difference (MD) and the respective 95\% confidence intervals in pulse wave velocity (PWV) before/after treatment from eligible studies. The size of the squares corresponds to the weight of each study. The diamonds and their width represent the pooled weighted MD and the 95\% CI, respectively. DPP-4: dipeptidyl peptidase-4; GLP-1 RAs: glucagon-like peptide-1 receptor agonists; SGLT-2: sodium-glucose cotransporter-2.

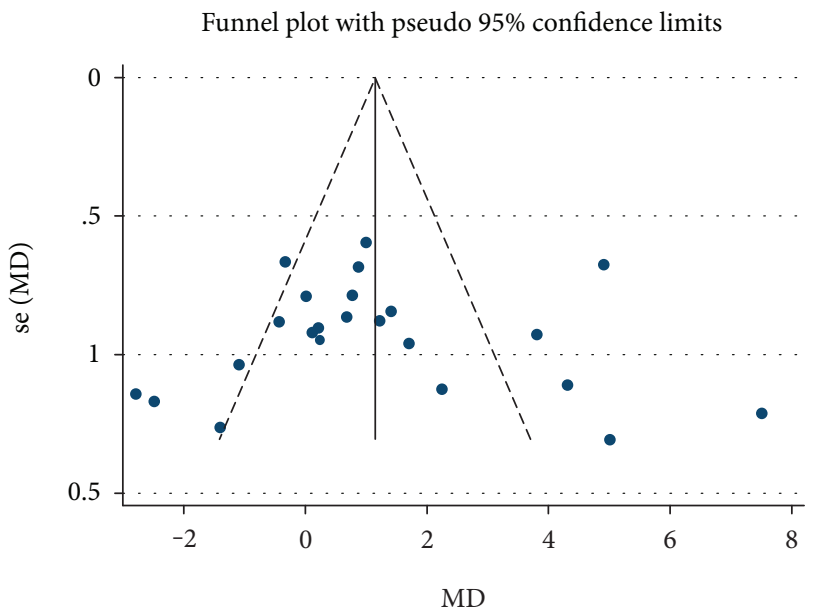

FIGURE 4: Funnel plot and assessment of publication bias. Funnel plot with 95\% pseudoconfidence intervals of the effect size and its standard error for studies assessing the effects of newer antidiabetic drugs on the primary endpoint endothelial function. Large studies appear toward the top of the graph and tend to cluster near the mean effect size. Smaller studies appear toward the bottom of the graph and (since there is more sampling variation in effect size estimates in the smaller studies) will be dispersed across a range of values. The symmetric distribution of studies about the combined effect size indicates the absence of publication bias.
The limitations of the existing studies in the field should be noted. The published evidence is modest and mainly based on small-sized randomized clinical studies, some of which were uncontrolled. Furthermore, studies were significantly heterogeneous, and therefore, the results of the present meta-analysis should be interpreted cautiously. The number of the published studies in this field did not allow for subgroup analysis per drug class or for between-agent comparisons within the same drug class. Furthermore, based on the published literature, we cannot conclude whether beneficial effects of SGLT-2 inhibitors, DDP-4 inhibitors, and GLP-1 RAs are due to direct glucose-lowering effects or to indirect effects driven by the modulation of other cardiovascular risk factors such as body weight loss and arterial blood pressure modification $[8-11,57]$. More data is also warranted for the effect of SGLT-2 inhibitors on arterial stiffness as well as endothelial function as there are limited published studies reporting on their effects.

\section{Conclusion}

In conclusion, the present meta-analysis suggests that the published literature in the field of newer antidiabetic drugs and vascular function is of modest quality and characterized by significant heterogeneity among studies. Overall, both DPP-4 inhibitors and GLP-1 RAs were shown to significantly 
decrease PWV without affecting FMD. In contrast, SGLT-2 inhibitors significantly improved FMD, but concrete data on their effects on PWV is still missing. Whether these distinct properties of newer antidiabetic drugs, in relation to their effects on endothelial function and arterial stiffness, may explain their differential effects on CVD risk remains to be elucidated in future studies.

\section{Conflicts of Interest}

The authors declare that they have no conflicts of interest.

\section{References}

[1] J. E. Shaw, R. A. Sicree, and P. Z. Zimmet, "Global estimates of the prevalence of diabetes for 2010 and 2030," Diabetes Research and Clinical Practice, vol. 87, no. 1, pp. 4-14, 2010.

[2] The Emerging Risk Factors Collaboration, "Diabetes mellitus, fasting blood glucose concentration, and risk of vascular disease: a collaborative meta-analysis of 102 prospective studies," The Lancet, vol. 375, no. 9733, pp. 2215-2222, 2010.

[3] C. C. Low Wang, C. N. Hess, W. R. Hiatt, and A. B. Goldfine, "Clinical update: cardiovascular disease in diabetes mellitus. Atherosclerotic cardiovascular disease and heart failure in type 2 diabetes mellitus - mechanisms, management and clinical considerations," Circulation, vol. 133, no. 24, pp. 2459-2502, 2016.

[4] A. S. Antonopoulos, G. Siasos, and D. Tousoulis, "Microangiopathy, arterial stiffness, and risk stratification in patients with type 2 diabetes," JAMA Cardiology, vol. 2, no. 7, pp. 820-821, 2017.

[5] A. S. Antonopoulos, M. Margaritis, P. Coutinho et al., "Adiponectin as a link between type 2 diabetes and vascular NADPH oxidase activity in the human arterial wall: the regulatory role of perivascular adipose tissue," Diabetes, vol. 64, no. 6, pp. 2207-2219, 2015.

[6] S. E. Nissen and K. Wolski, "Effect of rosiglitazone on the risk of myocardial infarction and death from cardiovascular causes," The New England Journal of Medicine, vol. 356, no. 24, pp. 2457-2471, 2007.

[7] R. R. Holman, H. Sourij, and R. M. Califf, "Cardiovascular outcome trials of glucose-lowering drugs or strategies in type 2 diabetes," Lancet, vol. 383, no. 9933, pp. 2008-2017, 2014.

[8] B. Zinman, C. Wanner, J. M. Lachin et al., "Empagliflozin, cardiovascular outcomes, and mortality in type 2 diabetes," New England Journal of Medicine, vol. 373, no. 22, pp. 2117-2128, 2015.

[9] S. P. Marso, G. H. Daniels, K. Brown-Frandsen et al., "Liraglutide and cardiovascular outcomes in type 2 diabetes," New England Journal of Medicine, vol. 375, no. 4, pp. 311-322, 2016.

[10] B. Neal, V. Perkovic, K. W. Mahaffey et al., "Canagliflozin and cardiovascular and renal events in type 2 diabetes," New England Journal of Medicine, vol. 377, no. 7, pp. 644-657, 2017.

[11] S. P. Marso, S. C. Bain, A. Consoli et al., "Semaglutide and cardiovascular outcomes in patients with type 2 diabetes," The New England Journal of Medicine, vol. 375, no. 19, pp. 18341844, 2016.

[12] M. A. Pfeffer, B. Claggett, R. Diaz et al., "Lixisenatide in patients with type 2 diabetes and acute coronary syndrome,"
The New England Journal of Medicine, vol. 373, no. 23, pp. 2247-2257, 2015.

[13] R. R. Holman, M. A. Bethel, R. J. Mentz et al., "Effects of onceweekly exenatide on cardiovascular outcomes in type 2 diabetes," The New England Journal of Medicine, vol. 377, no. 13, pp. 1228-1239, 2017.

[14] W. B. White, C. P. Cannon, S. R. Heller et al., "Alogliptin after acute coronary syndrome in patients with type 2 diabetes," The New England Journal of Medicine, vol. 369, no. 14, pp. 13271335, 2013.

[15] J. B. Green, M. A. Bethel, P. W. Armstrong et al., "Effect of sitagliptin on cardiovascular outcomes in type 2 diabetes," New England Journal of Medicine, vol. 373, no. 3, pp. 232-242, 2015.

[16] B. M. Scirica, D. L. Bhatt, E. Braunwald et al., "Saxagliptin and cardiovascular outcomes in patients with type 2 diabetes mellitus," New England Journal of Medicine, vol. 369, no. 14, pp. 1317-1326, 2013.

[17] A. S. Antonopoulos, F. Sanna, N. Sabharwal et al., "Detecting human coronary inflammation by imaging perivascular fat," Science Translational Medicine, vol. 9, no. 398, article eaal2658, 2017.

[18] A. S. Antonopoulos and D. Tousoulis, "The molecular mechanisms of obesity paradox," Cardiovascular Research, vol. 113, no. 9, pp. 1074-1086, 2017.

[19] A. S. Antonopoulos, G. Siasos, T. Konsola et al., "Arterial wall elastic properties and endothelial dysfunction in the diabetic foot syndrome in patients with type 2 diabetes," Diabetes Care, vol. 38, no. 11, pp. e180-e181, 2015.

[20] C. Antoniades, C. Bakogiannis, P. Leeson et al., "Rapid, direct effects of statin treatment on arterial redox state and nitric oxide bioavailability in human atherosclerosis via tetrahydrobiopterin-mediated endothelial nitric oxide synthase coupling," Circulation, vol. 124, no. 3, pp. 335345, 2011.

[21] C. Antoniades, C. Bakogiannis, D. Tousoulis et al., "Preoperative atorvastatin treatment in CABG patients rapidly improves vein graft redox state by inhibition of Racl and NADPHoxidase activity," Circulation, vol. 122, no. 11, Supplement 1, pp. S66-S73, 2010.

[22] S. P. Hozo, B. Djulbegovic, and I. Hozo, "Estimating the mean and variance from the median, range, and the size of a sample," BMC Medical Research Methodology, vol. 5, no. 1, p. 13, 2005.

[23] for the PROLOGUE Study Investigators, T. Maruhashi, Y. Higashi et al., "Long-term effect of sitagliptin on endothelial function in type 2 diabetes: a sub-analysis of the PROLOGUE study," Cardiovascular Diabetology, vol. 15, no. 1, 2016.

[24] K. Nakamura, H. Oe, H. Kihara et al., "DPP-4 inhibitor and alpha-glucosidase inhibitor equally improve endothelial function in patients with type 2 diabetes: EDGE study," Cardiovascular Diabetology, vol. 13, no. 1, p. 110, 2014.

[25] K. Suzuki, K. Watanabe, T. Suzuki et al., "Sitagliptin improves vascular endothelial function in Japanese type 2 diabetes patients without cardiovascular disease," Journal of Diabetes Mellitus, vol. 2, no. 3, pp. 338-345, 2012.

[26] T. Jax, A. Stirban, A. Terjung et al., "A randomised, active- and placebo-controlled, three-period crossover trial to investigate short-term effects of the dipeptidyl peptidase- 4 inhibitor linagliptin on macro- and microvascular endothelial function in type 2 diabetes," Cardiovascular Diabetology, vol. 16, no. 1, p. 13, 2017. 
[27] X. H. Wang, L. N. Han, Y. R. Yu et al., "Effects of GLP-1 agonist exenatide on cardiac diastolic function and vascular endothelial function in diabetic patients," Sichuan Da Xue Xue Bao. Yi Xue Ban, vol. 46, no. 4, pp. 586-590, 2015.

[28] S. H. Downs and N. Black, "The feasibility of creating a checklist for the assessment of the methodological quality both of randomised and non-randomised studies of health care interventions," Journal of Epidemiology and Community Health, vol. 52, no. 6, pp. 377-384, 1998.

[29] J. Matsubara, S. Sugiyama, E. Akiyama et al., "Dipeptidyl peptidase- 4 inhibitor, sitagliptin, improves endothelial dysfunction in association with its anti-inflammatory effects in patients with coronary artery disease and uncontrolled diabetes," Circulation Journal, vol. 77, no. 5, pp. 1337-1344, 2013.

[30] K. Striepe, A. Jumar, C. Ott et al., "Effects of the selective sodium-glucose cotransporter 2 inhibitor empagliflozin on vascular function and central hemodynamics in patients with type 2 diabetes mellitus," Circulation, vol. 136, no. 12, pp. 1167-1169, 2017.

[31] M. Ayaori, N. Iwakami, H. Uto-Kondo et al., "Dipeptidyl peptidase- 4 inhibitors attenuate endothelial function as evaluated by flow-mediated vasodilatation in type 2 diabetic patients," Journal of the American Heart Association, vol. 2, no. 1, article e003277, 2013.

[32] D. Baltzis, J. R. Dushay, J. Loader et al., "Effect of linagliptin on vascular function: a randomized, placebo-controlled study," The Journal of Clinical Endocrinology and Metabolism, vol. 101, no. 11, pp. 4205-4213, 2016.

[33] S. A. de Boer, H. J. L. Heerspink, L. E. Juárez Orozco et al., "Effect of linagliptin on pulse wave velocity in early type 2 diabetes: a randomized, double-blind, controlled 26-week trial (RELEASE)," Diabetes, Obesity and Metabolism, vol. 19, no. 8, pp. 1147-1154, 2017.

[34] R. Dell'Oro, A. Maloberti, F. Nicoli et al., "Long-term saxagliptin treatment improves endothelial function but not pulse wave velocity and intima-media thickness in type 2 diabetic patients," High Blood Pressure \& Cardiovascular Prevention, vol. 24, no. 4, pp. 393-400, 2017.

[35] E. Gurkan, I. Tarkun, T. Sahin, B. Cetinarslan, and Z. Canturk, "Evaluation of exenatide versus insulin glargine for the impact on endothelial functions and cardiovascular risk markers," Diabetes Research and Clinical Practice, vol. 106, no. 3, pp. 567-575, 2014.

[36] C. Irace, S. de Luca, E. Shehaj et al., "Exenatide improves endothelial function assessed by flow mediated dilation technique in subjects with type 2 diabetes: results from an observational research," Diabetes \& Vascular Disease Research, vol. 10, no. 1, pp. 72-77, 2012.

[37] G. Kim, S. Oh, S. M. Jin, K. Y. Hur, J. H. Kim, and M. K. Lee, "The efficacy and safety of adding either vildagliptin or glimepiride to ongoing metformin therapy in patients with type 2 diabetes mellitus," Expert Opinion on Pharmacotherapy, vol. 18, no. 12, pp. 1179-1186, 2017.

[38] N. Kitao, H. Miyoshi, T. Furumoto et al., "The effects of vildagliptin compared with metformin on vascular endothelial function and metabolic parameters: a randomized, controlled trial (Sapporo Athero-Incretin Study 3)," Cardiovascular Diabetology, vol. 16, no. 1, p. 125, 2017.

[39] V. Lambadiari, G. Pavlidis, F. Kousathana et al., "Effects of 6-month treatment with the glucagon like peptide-1 analogue liraglutide on arterial stiffness, left ventricular myocardial deformation and oxidative stress in subjects with newly diagnosed type 2 diabetes," Cardiovascular Diabetology, vol. 17, no. 1, p. 8, 2018.

[40] M. Leung, D. Y. Leung, and V. W. Wong, "Effects of dipeptidyl peptidase- 4 inhibitors on cardiac and endothelial function in type 2 diabetes mellitus: a pilot study," Diabetes \& Vascular Disease Research, vol. 13, no. 3, pp. 236243, 2016.

[41] F. Li, J. Chen, F. Leng, Z. Lu, and Y. Ling, "Effect of saxagliptin on circulating endothelial progenitor cells and endothelial function in newly diagnosed type 2 diabetic patients," Experimental and Clinical Endocrinology \& Diabetes, vol. 125, no. 6, pp. 400-407, 2017.

[42] H. Nomoto, H. Miyoshi, T. Furumoto et al., "A randomized controlled trial comparing the effects of sitagliptin and glimepiride on endothelial function and metabolic parameters: Sapporo Athero-Incretin Study 1 (SAIS1)," PLoS One, vol. 11, no. 10, p. e0164255, 2016.

[43] H. Nomoto, H. Miyoshi, T. Furumoto et al., "A comparison of the effects of the GLP-1 analogue liraglutide and insulin glargine on endothelial function and metabolic parameters: a randomized, controlled trial Sapporo Athero-Incretin Study 2 (SAIS2)," PLoS One, vol. 10, no. 8, p. e0135854, 2015.

[44] F. Shigiyama, N. Kumashiro, M. Miyagi et al., "Effectiveness of dapagliflozin on vascular endothelial function and glycemic control in patients with early-stage type 2 diabetes mellitus: DEFENCE study," Cardiovascular Diabetology, vol. 16, no. 1, p. 84, 2017.

[45] F. Shigiyama, N. Kumashiro, M. Miyagi et al., "Linagliptin improves endothelial function in patients with type 2 diabetes: a randomized study of linagliptin effectiveness on endothelial function," Journal of Diabetes Investigation, vol. 8, no. 3, pp. 330-340, 2017.

[46] A. Solini, L. Giannini, M. Seghieri et al., "Dapagliflozin acutely improves endothelial dysfunction, reduces aortic stiffness and renal resistive index in type 2 diabetic patients: a pilot study," Cardiovascular Diabetology, vol. 16, no. 1, p. 138, 2017.

[47] M. E. Widlansky, V. K. Puppala, T. M. Suboc et al., "Impact of DPP-4 inhibition on acute and chronic endothelial function in humans with type 2 diabetes on background metformin therapy," Vascular Medicine, vol. 22, no. 3, pp. 189-196, 2017.

[48] I. Zografou, C. Sampanis, E. Gkaliagkousi et al., "Effect of vildagliptin on hsCRP and arterial stiffness in patients with type 2 diabetes mellitus," Hormones, vol. 14, no. 1, pp. 118-125, 2015.

[49] L. Duvnjak and K. Blaslov, "Dipeptidyl peptidase-4 inhibitors improve arterial stiffness, blood pressure, lipid profile and inflammation parameters in patients with type 2 diabetes mellitus," Diabetology and Metabolic Syndrome, vol. 8, no. 1, p. 26, 2016.

[50] J. Y. Hong, K. Y. Park, B. J. Kim, W. M. Hwang, D. H. Kim, and D. M. Lim, "Effects of short-term exenatide treatment on regional fat distribution, glycated hemoglobin levels, and aortic pulse wave velocity of obese type 2 diabetes mellitus patients," Endocrinology and Metabolism, vol. 31, no. 1, pp. 80-85, 2016.

[51] N. D. Hopkins, D. J. Cuthbertson, G. J. Kemp et al., "Effects of 6 months glucagon-like peptide- 1 receptor agonist treatment on endothelial function in type 2 diabetes mellitus patients," Diabetes, Obesity \& Metabolism, vol. 15, no. 8, pp. 770-773, 2013. 
[52] S. Ida, K. Murata, K. Betou et al., "Effect of trelagliptin on vascular endothelial functions and serum adiponectin level in patients with type 2 diabetes: a preliminary single-arm prospective pilot study," Cardiovascular Diabetology, vol. 15, no. 1, p. 153, 2016.

[53] Y. Kubota, M. Miyamoto, G. Takagi et al., "The dipeptidyl peptidase-4 inhibitor sitagliptin improves vascular endothelial function in type 2 diabetes," Journal of Korean Medical Science, vol. 27, no. 11, pp. 1364-1370, 2012.

[54] G. Savarese, C. D'Amore, M. Federici et al., "Effects of dipeptidyl peptidase 4 inhibitors and sodium-glucose linked cotransporter- 2 inhibitors on cardiovascular events in patients with type 2 diabetes mellitus: a meta-analysis," International Journal of Cardiology, vol. 220, pp. 595-601, 2016.

[55] N. Katsiki, D. P. Mikhailidis, and M. J. Theodorakis, "Sodiumglucose cotransporter 2 inhibitors (SGLT2i): their role in cardiometabolic risk management," Current Pharmaceutical Design, vol. 23, no. 10, pp. 1522-1532, 2017.

[56] K. B. Margulies, K. J. Anstrom, A. F. Hernandez et al., “GLP-1 agonist therapy for advanced heart failure with reduced ejection fraction: design and rationale for the functional impact of GLP-1 for heart failure treatment study," Circulation. Heart Failure, vol. 7, no. 4, pp. 673-679, 2014.

[57] X. Zhang and Q. Zhao, "Effects of dipeptidyl peptidase-4 inhibitors on blood pressure in patients with type 2 diabetes: a systematic review and meta-analysis," Journal of Hypertension, vol. 34, no. 2, pp. 167-175, 2016. 


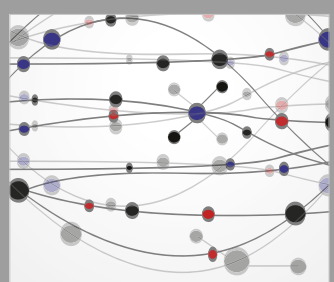

The Scientific World Journal
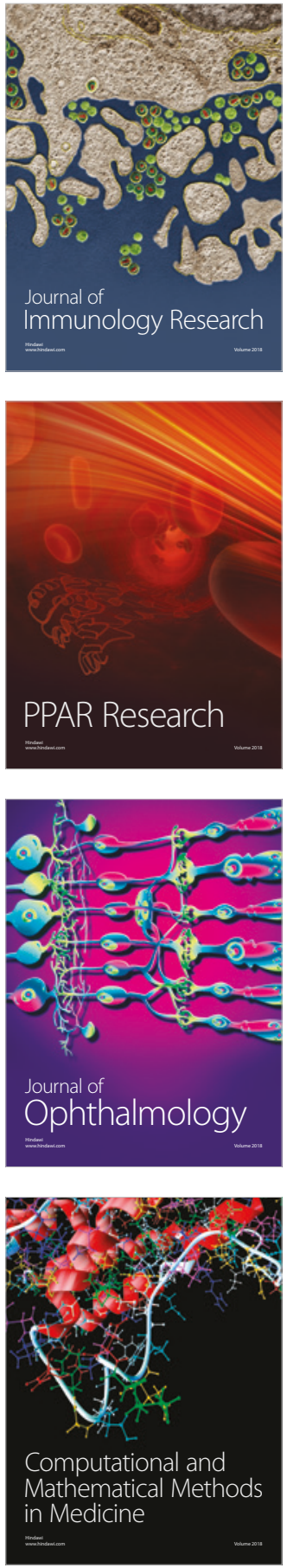

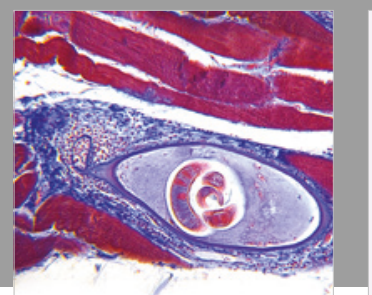

Gastroenterology Research and Practice

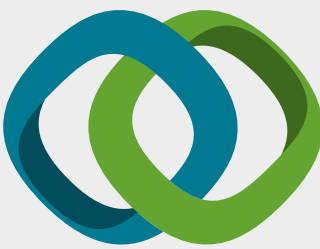

\section{Hindawi}

Submit your manuscripts at

www.hindawi.com
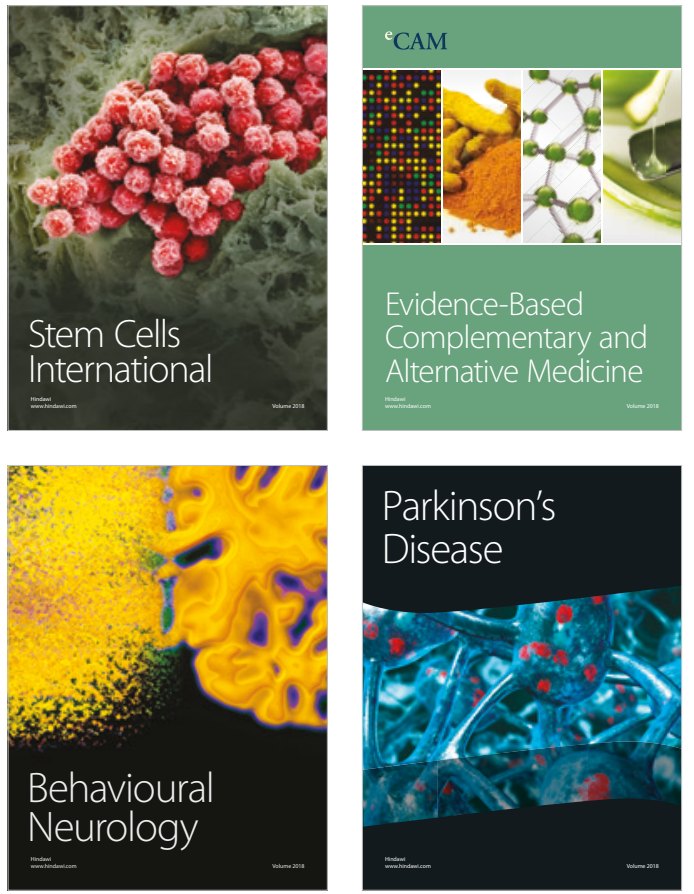

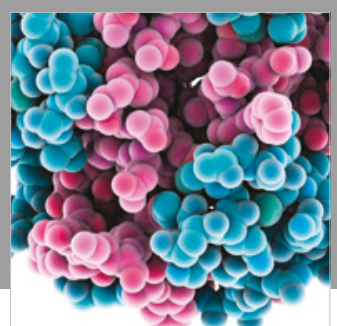

ournal of

Diabetes Research

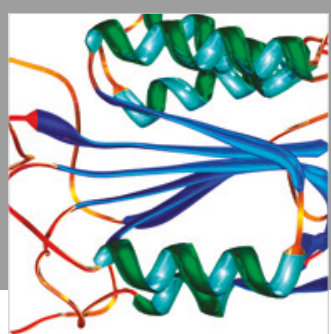

Disease Markers
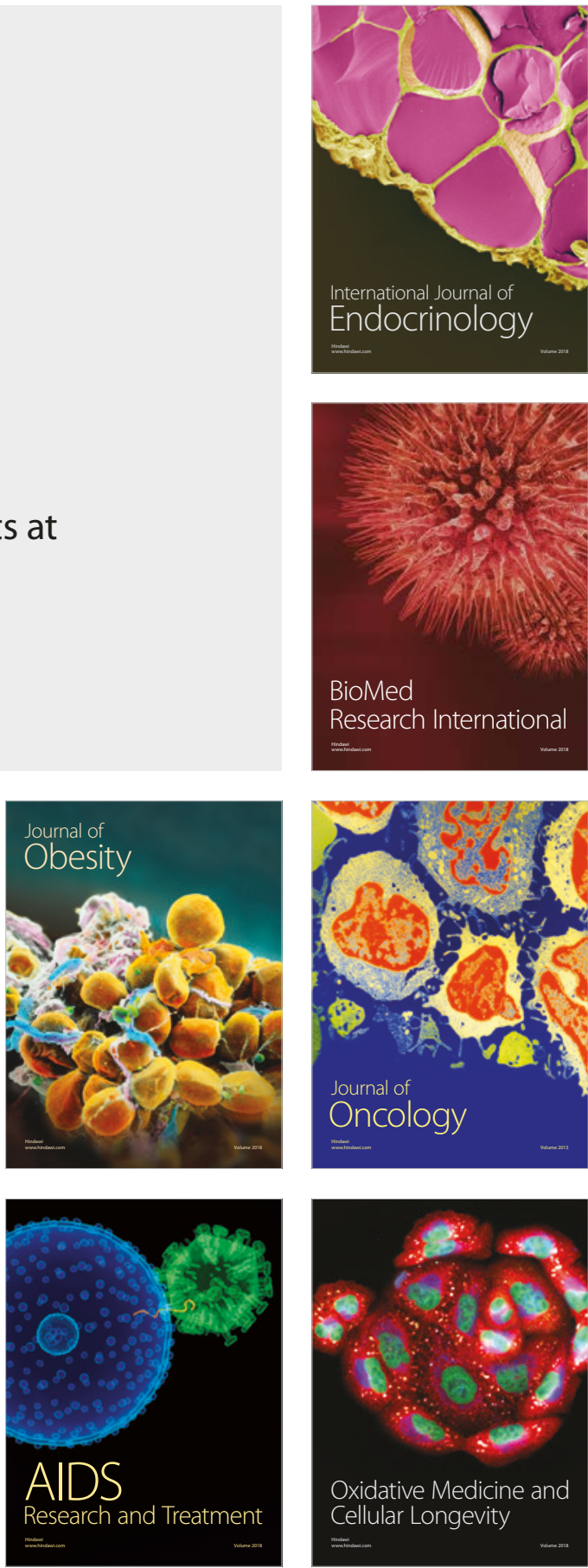